\title{
A CHARACTERIZATION OF HELICAL POLYNOMIAL CURVES OF ANY DEGREE
}

\author{
J. MONTERDE
}

\begin{abstract}
We give a full characterization of helical polynomial curves of any degree and a simple way to construct them. Existing results about Hermite interpolation are revisited. A simple method to select the best quintic interpolant among all possible solutions is suggested.
\end{abstract}

\section{INTRODUCTION}

The notion of helical polynomial curves, i.e, polynomial curves which made a constant angle with a fixed line in space, have been studied by different authors. Let us cite the papers $[6,7,8]$ where the main results about the cubical and quintic cases are stablished. In [6] the authors gives a necessary condition a polynomial curve must satisfy in order to be a helix. The condition is expressed in terms of its hodograph, i.e., its derivative. If a polynomial curve, $\alpha$, is a helix then its hodograph, $\alpha^{\prime}$, must be Pythagorean, i.e., $\left\|\alpha^{\prime}\right\|^{2}$ is a perfect square of a polynomial. Moreover, this condition is sufficient in the cubical case: all Pythagorean hodograph $(\mathrm{PH})$ cubical curves are helices. In the same paper it is also stated that not only $\alpha^{\prime}$ must be Pythagorean, but also $\alpha^{\prime} \wedge \alpha^{\prime \prime}$. Following the same ideas, in [2] it is proved that both conditions are sufficient in the quintic case. Unfortunately, this characterization is no longer true for higher degrees. It is possible to construct examples of polynomial curves of degree 7 verifying both conditions but being not a helix.

The aim of this paper is just to show how a simple geometric trick can clarify the proofs of some previous results and to simplify the needed computations to solve some related problems as for instance, the Hermite problem using helical polynomial curves.

The Dietz-Hoschek-Jüttler theorem (see [3] or [4]) relates the Pythagorean condition to the Hopf map $H: \mathbb{C}^{2} \rightarrow \mathbb{R}^{3}$. A polynomial curve $\alpha$ is Pythagorean hodograph curve if and only if there exist $z_{1}, z_{2}$ such that $\alpha^{\prime}=H\left(z_{1}, z_{2}\right)$ where $z_{1}, z_{2}$ are two polynomial complex functions.

The geometric trick is based on the following facts: first, the normalized Hopf map is related to the stereographic projection (the usual stereographic

Date: November 27, 2007.

2000 Mathematics Subject Classification. Primary 53A04; Secondary 53C40.

Key words and phrases. generalized polynomial helices, Pythagorean hodograph curves. 
projection from the two dimensional unit sphere, $S^{2}$, into the complex plane, not the generalized stereographic projection from the three-dimensional projective space, using homogeneous coordinates, to $S^{2}$, as it appears in Remark 1 of [6], or in Remark 2 of [7]); second, the tangent indicatrix of a helical curve describes a circle on the unit sphere and, finally, the image by the stereographic projection of a circle on the unit sphere is a line-or-circle in the complex plane.

Joining up these arguments, we obtain a full characterization of polynomial helices of any degree in terms of the quotient $\frac{z_{1}}{z_{2}}$. The condition is of geometric nature: $\alpha$ is an helix if and only if $\frac{z_{1}}{z_{2}}$ is a rational parametrization of a piece of a line or a circle in $\mathbb{C}$ depending whether or not the vector $(0,0,1)$ belongs to the hodograph, respectively.

According to this approach, the already known results $([7,8])$ about Hermite interpolation using helical curves are revisited and their proofs simplified. Moreover, the classification into two classes of helical PH quintics introduced in [6] is also revisited, and a result concerning a sufficient condition on the quaternions generating a $\mathrm{PH}$ helical curve is introduced (see Cor. 2).

Finally, a simple method to choose the best among all possible general PH quintic helices solving the first order Hermite problem is suggested. In the cited references, the choice among all the possible solutions involves the computation of some integral, the energy associated with the rotationminimizing frame. Here we simply control the values of two parameters involved in our method (see Prop. 1).

\section{Spatial Pythagorean hodograph curves}

Initially, we will use the quaternion representation of spatial $\mathrm{PH}$ curves. Given a quaternion polynomial

$$
\mathcal{A}(t)=u(t)+\mathbf{i} q(t)+\mathbf{j} p(t)+\mathbf{k} v(t),
$$

the product

$$
\alpha^{\prime}(t)=\mathcal{A}(t) \mathbf{k} \mathcal{A}^{*}(t)
$$

defines (the asterisk means quaternionic conjugate) a spatial Pythagorean hodograph, $\alpha^{\prime}$, whose components are

$$
\begin{aligned}
& x^{\prime}=2(u p+v q), \\
& y^{\prime}=2(v p-u q), \\
& z^{\prime}=u^{2}+v^{2}-p^{2}-q^{2},
\end{aligned}
$$

and such that $\left\|\alpha^{\prime}\right\|^{2}=\left(x^{\prime}\right)^{2}+\left(y^{\prime}\right)^{2}+\left(z^{\prime}\right)^{2}=\left(u^{2}+v^{2}+p^{2}+q^{2}\right)^{2}$.

In terms of the Hopf map (see [4], theorem 4.2)

$$
H: \mathbb{C}^{2} \rightarrow \mathbb{R}^{3}
$$

defined by $H\left(z_{1}, z_{2}\right)=\left(2 z_{1} \bar{z}_{2},\left|z_{1}\right|^{2}-\left|z_{2}\right|^{2}\right)$, and taking

$$
z_{1}(t)=u(t)+\mathbf{i} v(t), \quad z_{2}(t)=p(t)+\mathbf{i} q(t),
$$


the derivative of the curve can be written as

$$
\alpha^{\prime}(t)=H\left(z_{1}(t), z_{2}(t)\right) .
$$

Remark 1. We have followed the notations in [4], section 4, in particular the use of the basis element $\mathbf{k}$, in order to get the expression $\left(2 z_{1} \bar{z}_{2},\left|z_{1}\right|^{2}-\right.$ $\left|z_{2}\right|^{2}$ ) for the definition of the Hopf map.

The choice of the basis element $\mathbf{k}$ between $\mathcal{A}(t)$ and $\mathcal{A}^{*}(t)$ has no special significance as it is said in [5], page 411: choosing $\mathbf{i}$, as it is done in [6], or $\mathbf{j}$ instead leads to permutations of $x^{\prime}, y^{\prime}, z^{\prime}$ and $u, v, p, q$.

The order of symbols $u, v, p, q$ in $\mathcal{A}(t)$ is the one leading to alphabetical order in $z_{1}$ and $z_{2}$ (Formula 2.2). Note that all these choices lead to expressions of $x^{\prime}=u p+v q$ and $y^{\prime}=v p-u q$ where the factors $p$ and $q$ are swapped from the paper [6].

Theorem 1. A Pythagorean polynomial curve $\alpha$ with $\alpha^{\prime}(t)=H\left(z_{1}(t), z_{2}(t)\right)$ is a helix if and only if the curve in the complex plane $t \rightarrow \frac{z_{1}(t)}{z_{2}(t)}$ traces a line-or-circle.

Proof. A curve $\alpha$ is a general helix if and only if its tangent indicatrix is a subset of a circle on the unit sphere $S^{2}$.

Let us define the normalized Hopf map $H_{n o r}: \mathbb{C}^{2} \rightarrow S^{2}$ by

$$
H_{\text {nor }}\left(z_{1}, z_{2}\right)=\frac{1}{\left|z_{1}\right|^{2}+\left|z_{2}\right|^{2}}\left(2 z_{1} \bar{z}_{2},\left|z_{1}\right|^{2}-\left|z_{2}\right|^{2}\right) .
$$

If $\alpha^{\prime}(t)=H\left(z_{1}(t), z_{2}(t)\right)$, then its tangent indicatrix is

$$
\frac{\alpha^{\prime}(t)}{\left\|\alpha^{\prime}(t)\right\|}=H_{n o r}\left(z_{1}(t), z_{2}(t)\right) \text {. }
$$

Notice that $H_{\text {nor }}\left(z_{1}, z_{2}\right)=H_{\text {nor }}\left(\frac{z_{1}}{z_{2}}, 1\right)$ (See [1], section 4.3), and that for any $z \in \mathbb{C}, H_{\text {nor }}(z, 1)=p_{\text {st }}^{-1}(z)$ where $p_{\text {st }}: S^{2} \rightarrow \mathbb{C} \cup\{\infty\}$ denotes the extended stereographic projection from the north pole of the whole unit sphere to the extended complex plane with the assumption $p_{s t}(0,0,1)=\infty$.

Therefore, $\alpha$ is a general helix if and only if $p_{s t}^{-1}\left(\frac{z_{1}(t)}{z_{2}(t)}\right) \in S^{2}$ belongs to a circle in the unit sphere. But the image by the stereographic projection of a circle in the unit sphere is a line-or-circle in the extended complex plane (see Proposition 7.23 in [9]).

Corollary 1. A Pythagorean polynomial curve $\alpha$ with $\alpha^{\prime}(t)=H\left(z_{1}(t), z_{2}(t)\right)$ with $(0,0,1)$ in the trace of tangent indicatrix is a helix if and only if the curve in the complex plane $t \rightarrow \frac{z_{1}(t)}{z_{2}(t)}$ traces a piece of a straight line.

Proof. Recall that a circle on the unit sphere passes trough the north pole if and only if its image by the extended stereographic projection is a straight line of the complex plane. 


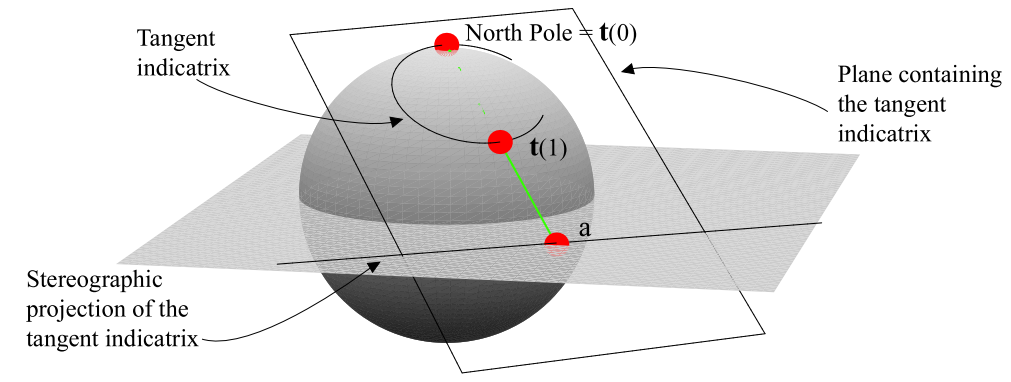

Figure I. Representation of the tangent indicatrix of a helical curve and of its stereographic projection.

We prove now the following result which, in the quadratic case, is a particular case of a result already proved in Proposition 1 in [6].

Corollary 2. Let $\mathcal{A}_{0}$ and $\mathcal{A}_{n}$ be two quaternionic numbers and let $\mathcal{A}_{i}=$ $\left(1-c_{i}\right) \mathcal{A}_{0}+c_{i} \mathcal{A}_{n}, c_{i} \in \mathbb{R}$, for all $i=1, \ldots, n-1$. If $\mathcal{A}(t)$ is the quaternionic Bézier curve defined by $\left\{\mathcal{A}_{i}\right\}_{i=0}^{n}$, then $\alpha^{\prime}(t)=\mathcal{A}(t) \mathbf{k} \mathcal{A}(t)^{*}$ defines a helical PH curve.

Proof. Let us write $\mathcal{A}_{i}=\left(1-c_{i}\right) \mathcal{A}_{0}+c_{i} \mathcal{A}_{n}$ for all $i=0,1, \ldots, n$ where $c_{i} \in \mathbb{R}, c_{0}=0$ and $c_{n}=1$. Then

$$
\mathcal{A}(t)=\sum_{i=0}^{n} B_{i}^{n}(t) \mathcal{A}_{i}=(1-m(t)) \mathcal{A}_{0}+m(t) \mathcal{A}_{n},
$$

where

$$
m(t)=\sum_{i=0}^{n} B_{i}^{n}(t) c_{i}
$$

Note that $\mathcal{A}(t)$ is a parametrization (not necessarily linear) of a piece of the quaternionic straight line passing through $\mathcal{A}_{0}$ and $\mathcal{A}_{n}$.

Let us suppose that $\mathcal{A}_{0}=u_{0}+\mathbf{i} q_{0}+\mathbf{j} p_{0}+\mathbf{k} v_{0}$ and $\mathcal{A}_{n}=u_{n}+\mathbf{i} q_{n}+\mathbf{j} p_{n}+\mathbf{k} v_{n}$. Then $\alpha^{\prime}(t)=\mathcal{A}(t) \mathbf{k} \mathcal{A}(t)^{*}=H\left(z_{1}(t), z_{2}(t)\right)$ where

$$
\left\{\begin{array}{c}
z_{1}(t)=\left(\left(1-m(t) u_{0}+m(t) u_{n}\right)+\mathbf{i}\left(\left(1-m(t) v_{0}+m(t) v_{n}\right)\right.\right. \\
=\left(u_{n}-u_{0}+\mathbf{i}\left(v_{n}-v_{0}\right)\right) m(t)+\left(u_{0}+\mathbf{i} v_{0}\right) \\
z_{2}(t)=\left(\left(1-m(t) p_{0}+m(t) p_{n}\right)+\mathbf{i}\left(\left(1-m(t) q_{0}+m(t) q_{n}\right)\right.\right. \\
=\left(p_{n}-p_{0}+\mathbf{i}\left(q_{n}-q_{0}\right)\right) m(t)+\left(p_{0}+\mathbf{i} q_{0}\right) .
\end{array}\right.
$$

Let us write

$$
z_{1}(t)=a m(t)+b, z_{2}(t)=c m(t)+d,
$$

for some $a, b, c, d \in \mathbb{C}$. Note that if $a d=c b$ then $\frac{z_{1}(t)}{z_{2}(t)}$ is a constant. So, we can suppose that $a d \neq c b$. 
Let us consider the Moebius transformation $f(z)=\frac{a z+b}{c z+d}$. Note that

$$
\frac{z_{1}(t)}{z_{2}(t)}=\frac{a m(t)+b}{c m(t)+d}=f(m(t))
$$

The map $t \rightarrow m(t)$ parametrizes a piece the real axis in the complex plane. Since the image under a Moebius transformation of a line is either a line or a circle (see Prop. 9.2 in [9]), then $\frac{z_{1}(t)}{z_{2}(t)}$ traces a line-or-circle. By Th. 1, the curve is a helical PH.

2.1. Examples. It is just a matter of computation to check that the two examples of quintic helices in [6] verify the condition written in the statement of Th. 1. As it is said in the cited reference, they are, respectively, examples of the two classes of helical PH quintics. We include here the definition of both classes for this paper to be self-contained: A PH curve, $\alpha(t)=(x(t), y(t), z(t))$, is monotone-helical if $x^{\prime}, y^{\prime}, z^{\prime}$ (and hence, also $\left.\sigma(t)=\sqrt{\left(x^{\prime}(t)\right)^{2}+\left(y^{\prime}(t)\right)^{2}+\left(z^{\prime}(t)\right)^{2}}\right)$ possess a non-constant common factor. In other case, this is, if $\operatorname{gcd}\left(x^{\prime}, y^{\prime}, z^{\prime}\right)=$ constant, the PH curve is called "a general helical PH curve".

2.1.1. Monotone-helical PH quintic example. The first example is defined by the four quadratic polynomials ${ }^{1}$

$u(t)=t^{2}-3 t, \quad v(t)=t^{2}-5 t+10, \quad p(t)=t^{2}-9 t+10, \quad q(t)=-2 t^{2}+3 t+5$.

Let us recall that for this case the polynomials $z_{1}$ and $z_{2}$ share a common factor corresponding to the root $1+2 \mathbf{i}$. So, we can simplify the quotient $\frac{z_{1}(t)}{z_{2}(t)}$ and to check that it belongs to the circumference with center $\left(\frac{3}{10}, \frac{1}{10}\right)$ and radius $r=\frac{\sqrt{2}}{2}$.

Moreover, let us see for this example which kind of rational parametrization of a straight line is $\frac{z_{1}(t)}{z_{2}(t)}$ when the hodograph is rotated in the space in such a way that the tangent vector at $t=0$ is $(0,0,1)$.

After a rotation with $y$-axis and angle $\arccos \left(\frac{1}{\sqrt{17}}\right)$ and a rotation with $x$-axis and angle $\arccos \left(\frac{\sqrt{17}}{9}\right)$ the hodograph curve is transformed into

$$
\alpha^{\prime}(t)=\left(5-2 t+t^{2}\right)\left(\frac{2 t(7 t-15)}{\sqrt{17}}, \frac{2 t(71 t-225)}{9 \sqrt{17}}, \frac{1}{9}\left(405-288 t+43 t^{2}\right)\right) .
$$

Now, it verifies $\overrightarrow{\mathbf{t}}(0)=\frac{\alpha^{\prime}(0)}{\left\|\alpha^{\prime}(0)\right\|}=(0,0,1)$.

Let us compute the image by the stereographic projection from the north pole of the tangent vector $\frac{\alpha^{\prime}(t)}{\left\|\alpha^{\prime}(t)\right\|}$. But, in order to avoid the singularity introduced by the stereographic projection at $t=0$ (recall that at $t=0$ the

\footnotetext{
${ }^{1}$ In order to keep our notations as it has been explained in Remark 1, in the next two examples, polynomials $p(t)$ and $q(t)$ are swapped from the original paper [6]
} 
tangent vector is the north pole where the stereographic projection is not defined) we will change $t$ by $\frac{1}{t}$.

$$
p_{s t}\left(\frac{\alpha^{\prime}\left(\frac{1}{t}\right)}{\left\|\alpha^{\prime}\left(\frac{1}{t}\right)\right\|}\right)=\frac{1}{10 \sqrt{17}}(63+71 \mathbf{i}-(135+225 \mathbf{i}) t) .
$$

2.1.2. General helical PH quintic example. The second example is defined by

$$
\begin{array}{ll}
u(t)=-19 t^{2}+12 t+5, & v(t)=-22 t^{2}+18 t+1, \\
p(t)=-31 t^{2}+24 t+3, & q(t)=15 t^{2}-12 t-1 .
\end{array}
$$

Now, $\frac{z_{1}(t)}{z_{2}(t)}$ belongs to the circumference with center $\left(\frac{3}{4},-\frac{15}{4}\right)$ and radius $r=\frac{13 \sqrt{2}}{4}$.

Again, let us see for this example which kind of rational parametrization of a straight line is $\frac{z_{1}(t)}{z_{2}(t)}$ when the hodograph is rotated in the space in such a way that the tangent vector at $t=0$ is $(0,0,1)$. Thanks to a rotation with $y$ axis and angle $\arccos \left(\frac{4}{\sqrt{65}}\right)$ and a rotation with $x$-axis and angle $\arccos \left(\frac{\sqrt{65}}{9}\right)$ the hodograph curve is transformed into

$$
\begin{gathered}
\alpha^{\prime}(t)=\left(\sqrt{\frac{13}{5}} t(7 t-6)\left(-14-30 t+49 t^{2}\right),\right. \\
\frac{2}{9} \sqrt{\frac{13}{5}} t(7 t-6)\left(-81-480 t+641 t^{2}\right), \\
\left.36+\frac{2}{9} t\left(1458+279 t-6834 t^{2}+4999 t^{3}\right)\right) .
\end{gathered}
$$

In this case, and again with the change $t \rightarrow \frac{1}{t}$,

$$
p_{s t}\left(\frac{\alpha^{\prime}\left(\frac{1}{t}\right)}{\left\|\alpha^{\prime}\left(\frac{1}{t}\right)\right\|}\right)=\frac{1}{13 \sqrt{65}(6 t-7)}\left(-(441+1282 \mathbf{i})+(270+960 \mathbf{i}) t+(126+162 \mathbf{i}) t^{2}\right) \text {. }
$$

The result is now a second degree rational parametrization of a straight line. If we see it as a rational Bézier curve, its control points are

$$
P_{0}=c\left(63, \frac{1282}{7}\right), \quad P_{1}=c\left(\frac{153}{2}, \frac{401}{2}\right), \quad P_{2}=c(45,160),
$$

with $c=\frac{1}{13 \sqrt{65}}$, and its weights are

$$
\omega_{0}=7, \quad \omega_{1}=4, \quad \omega_{2}=1 .
$$

Note that $P_{1}=\frac{7}{4} P_{0}+\left(1-\frac{7}{4}\right) P_{2}$. 
2.1.3. An example of degree 7. In the paper [2] we gave an example of a degree seven polynomial curve,

$$
\alpha(t)=\left(-3 t+t^{3}+\frac{t^{5}}{5}+\frac{t^{7}}{21}, 3 t^{2}-\frac{t^{4}}{2},-2 t^{3}\right),
$$

verifying that both $\alpha^{\prime}$ and $\alpha^{\prime} \wedge \alpha^{\prime \prime}$ were Pythagorean but still not being an helix. It is easy to check that in this case the quotient $\frac{z_{1}(t)}{z_{2}(t)}$ does not belong to a circumference nor a straight line.

\section{Construction of helical Curves}

3.1. Case $\frac{z_{1}}{z_{2}}$ belongs to a line. If $\frac{z_{1}(t)}{z_{2}(t)}$ belongs to a straight line then it can be written as

$$
\frac{z_{1}(t)}{z_{2}(t)}=a+\frac{m(t)}{n(t)} b, \quad a, b \in \mathbb{C},
$$

where $m(t)$ and $n(t)$ are real polynomials.

The hodograph of the associated helix can be computed using

$$
z_{1}(t)=a n(t)+b m(t), z_{2}(t)=n(t) .
$$

Therefore,

$$
\alpha^{\prime}=\left(2 a n^{2}+2 b m n,\left(|a|^{2}-1\right) n^{2}+(a \bar{b}+\bar{a} b) m n+|b|^{2} m^{2}\right),
$$

where we have suppressed any reference to the parameter $t$ and where the first two coordinates of $\alpha^{\prime}$ are written as a complex number.

Since the curvature and the torsion of a curve are geometric quantities, i.e., independent of the parametrization we can compute them supposing that

$$
\frac{z_{1}(t)}{z_{2}(t)}=a+t b, \quad a, b \in \mathbb{C} .
$$

In this case, it is just a matter of computation to check that

$$
\frac{\tau(t)}{\kappa(t)}=\frac{\operatorname{Im}(a \cdot \bar{b})}{|b|} \text {. }
$$

3.2. Case $\frac{z_{1}}{z_{2}}$ belongs to a circle. If $\frac{z_{1}(t)}{z_{2}(t)}=\frac{p_{x}}{q}+\mathbf{i} \frac{p_{y}}{q}$ belongs to a circle then there exist $c=c_{x}+\mathbf{i} c_{y} \in \mathbb{C}$ and $r \in \mathbb{R}$ such that

$$
\left|\frac{p_{x}+\mathbf{i} p_{y}}{q}-c\right|^{2}=r^{2} \text {. }
$$

As before, we can compute the ratio $\frac{\tau}{\kappa}$ supposing that

$$
\frac{z_{1}(t)}{z_{2}(t)}=c+r e^{\mathrm{i} t}, \quad c \in \mathbb{C}, r \in \mathbb{R} .
$$

In this case, it is again a matter of computation to check that

$$
\frac{\tau(t)}{\kappa(t)}=\frac{1+|c|^{2}-r^{2}}{2 r} .
$$


Moreover, note that the curve $\left(p_{x}(t)-q(t) c_{x}, p_{y}(t)-q(t) c_{y}\right)$ is a planar PHcurve. Therefore, we can apply the well known complex representation of a planar $\mathrm{PH}$-curve (see [5]) to the previous equation: there are polynomials $m(t), n(t)$ such that

$$
p_{x}-q c_{x}=m^{2}-n^{2}, \quad p_{y}-q c_{y}=2 m n, \quad r q=m^{2}+n^{2} .
$$

So,

$$
\frac{z_{1}(t)}{z_{2}(t)}=c+\frac{(m(t)+\mathbf{i} n(t))^{2}}{q(t)}=c+r \frac{m(t)+\mathbf{i} n(t)}{m(t)-\mathbf{i} n(t)} .
$$

In this case, the hodograph of the associated helix can be computed using

$$
z_{1}(t)=c(m(t)-\mathbf{i} n(t))+r(m(t)+\mathbf{i} n(t)), \quad z_{2}(t)=m(t)-\mathbf{i} n(t) .
$$

Putting $c=c_{x}+\mathbf{i} c_{y}$, suppressing any reference to the parameter $t$ and writing the first two coordinates of $\alpha^{\prime}$ as a complex number we get

$$
\begin{aligned}
\alpha^{\prime}=\left(2\left(c\left(m^{2}+n^{2}\right)+r(m+\mathbf{i} n)^{2}\right),\right. \\
\\
\left.\quad\left(m^{2}+n^{2}\right)\left(c_{y}^{2}+\left(c_{x}-r\right)^{2}-1\right)+4 r m\left(c_{x} m+c_{y} n\right)\right) .
\end{aligned}
$$

4. First-order Hermite helical interpolants Revisited. The CUBICAL CASE

In [8] the following Hermite interpolation problem is considered: Given end points $\mathbf{p}_{0}$ and $\mathbf{p}_{1}$ and end tangents $\overrightarrow{\mathbf{t}}_{0}$ and $\overrightarrow{\mathbf{t}}_{1}$ satisfying $\left(\overrightarrow{\mathbf{t}}_{0} \times\right.$ $\left.\overrightarrow{\mathbf{t}}_{1}\right) \cdot\left(\mathbf{p}_{1}-\mathbf{p}_{0}\right) \neq 0$, interpolate such data with a spatial PH cubic. Since PH cubics are helical curves all the machinery on such kind of curves can be used.

The problem is studied in the cited reference with full detail. A simple geometric condition is obtained to test the existence of two, one, or none, interpolants. As it is said there these conditions agree and extent previous results in [7].

The following theorem states the previous results for cubical interpolants. The statement is not exactly the one in [8].

Theorem 2. [8, 7] Let $0 \leq \theta_{0}, \theta_{1} \leq \pi$ be the angles of the unit tangents $\overrightarrow{\mathbf{t}}_{0}$ and $\overrightarrow{\mathbf{t}}_{1}$ with respect to the vector $\mathbf{p}_{1}-\mathbf{p}_{0}$, then a necessary condition for the existence of spatial PH cubics interpolants is that

$$
\overrightarrow{\mathbf{t}}_{0} \cdot \overrightarrow{\mathbf{t}}_{1} \leq \frac{3}{4} \cos \left(\theta_{0}+\theta_{1}\right)+\frac{1}{4} \text {. }
$$

Moreover,

$$
\left\{\begin{array}{l}
\text { a) if } \overrightarrow{\mathbf{t}}_{0} \cdot \overrightarrow{\mathbf{t}}_{1} \leq-\frac{1}{2} \quad \text { there is just one regular interpolant, } \\
\text { b) if } \overrightarrow{\mathbf{t}}_{0} \cdot \overrightarrow{\mathbf{t}}_{1}>-\frac{1}{2} \quad \text { there are }\left\{\begin{array}{l}
\text { two interpolants if } \quad \cos \theta_{0}>0 \\
\text { no interpolant if } \quad \cos \theta_{0} \leq 0
\end{array}\right.
\end{array}\right.
$$


The proof can be found in the cited references and, although it can be simplified at the new light shed by Th. 1 and Corollary 1, we have decided not to include it here. Nevertheless, we will recall how to compute the cubical interpolants, in the case they exist.

First of all, thanks to a translation, a rotation and a scale factor, we can reduce the Hermite problem to the following data:

End points $\mathbf{p}_{0}=(0,0,0)$ and $\mathbf{p}_{1}=\left(x_{p}, y_{p}, z_{p}\right) \in S^{2}$ and end tangents $\overrightarrow{\mathbf{t}}_{0}=(0,0,1)$ and $\overrightarrow{\mathbf{t}}_{1}=\left(x_{t}, y_{t}, z_{t}\right)$.

Since $\overrightarrow{\mathbf{t}}(0)=\overrightarrow{\mathbf{t}}_{0}=(0,0,1)$, and according to Corollary $1, \frac{z_{1}}{z_{2}}(t)$ must be a rational parametrization of a straight line such that $\frac{z_{1}}{z_{2}}(0)=\infty$. Moreover, for $\mathrm{PH}$ cubical curves the polynomials $z_{1}(t)$ and $z_{2}(t)$ are of degree 1 , then we can write the quotient as follows

$$
\frac{z_{1}}{z_{2}}(t)=\frac{1}{t} a+\left(1-\frac{1}{t}\right) b, \quad a, b \in \mathbb{C} .
$$

Equivalently,

$$
z_{1}(t)=\sqrt{3} m(a+(t-1) b), \quad z_{2}(t)=\sqrt{3} m t,
$$

where $m \in \mathbb{C}$. The factor $\sqrt{3}$ has been introduced to keep computations in the sequel as simple as possible.

From Eq. (4.3)

$$
a=\frac{z_{1}}{z_{2}}(1)=p_{s t}(\overrightarrow{\mathbf{t}}(1))=p_{s t}\left(\overrightarrow{\mathbf{t}}_{1}\right)=\frac{x_{t}+\mathbf{i} y_{t}}{1-z_{t}} .
$$

An easy computation shows that

$$
\begin{aligned}
\int_{0}^{1} 2 z_{1}(t) \bar{z}_{2}(t) d t & =|m|^{2}(3 a-b), \\
\int_{0}^{1}\left(\left|z_{1}(t)\right|^{2}-\left|z_{2}(t)\right|^{2}\right) d t & =|m|^{2}\left(3|a|^{2}-3 a \cdot b+|b|^{2}-1\right) .
\end{aligned}
$$

Therefore, imposing the condition $\alpha(1)=\mathbf{p}_{1}$ we get, from the first two coordinates,

$$
b=3 a-\frac{x_{p}+\mathbf{i} y_{p}}{|m|^{2}} .
$$

Finally, from the third coordinate of $\alpha(1)=\mathbf{p}_{1}$, we obtain a quadratic equation in the variable $|m|^{2}$. Up to non zero factors, it can be written as a biquadratic equation in the unique unknown $|m|$ :

$$
1-z_{p}^{2}-\left(3\left(a_{0} x_{p}+a_{1} y_{p}\right)+z_{p}\right)|m|^{2}+\left(3|a|^{2}-1\right)|m|^{4}=0,
$$

where $a=a_{0}+\mathbf{i} a_{1}, a_{0}, a_{1} \in \mathbb{R}$.

The existence or not of interpolants depends, firstly, on the non negativity of the discriminant of the biquadratic equation (4.6), and secondly, on the number of positive solutions. 


\section{First-order Hermite helical interpolants Revisited. The QUINTIC CASE}

In the paper [6], the authors study the use of quintic helices to solve the first order Hermite interpolation problem. If one wants to adjust not only the initial and final directions, but also their magnitudes we must to increase the degree of the polynomial curve.

As we have noticed before, in the cited reference two classes of quintic $\mathrm{PH}$ helical curves are described. We shall follow the same terminology for them, monotone and general, (see the first paragraph of Example 2.1 for definitions) and we will try to solve the Hermite problem within both classes.

We shall study the following Hermite interpolation problem in the space: find a quintic $\mathrm{PH}$ helical curve, $\alpha$, such that

$$
\alpha(0)=\mathbf{p}_{0}, \quad \alpha^{\prime}(0)=\mathbf{d}_{0} \quad \text { and } \quad \alpha(1)=\mathbf{p}_{1}, \quad \alpha^{\prime}(1)=\mathbf{d}_{1} .
$$

As before, thanks to a translation, a rotation and a scale factor, the problem can be reduced to

and

$$
\alpha(0)=(0,0,0), \quad \alpha^{\prime}(0)=(0,0,1)
$$

$$
\alpha(1)=\mathbf{q}_{1}=r_{q}\left(x_{q}, y_{q}, z_{q}\right), \quad \alpha^{\prime}(1)=\mathbf{v}_{1}=r_{v}\left(x_{v}, y_{v}, z_{v}\right),
$$

where $r_{q}, r_{v}$ are positive real numbers and $\left(x_{q}, y_{q}, z_{q}\right),\left(x_{v}, y_{v}, z_{v}\right) \in S^{2}$.

5.1. Using monotone-helical $\mathbf{P H}$ interpolants. We are looking for a helical curve, $\alpha$, as in the previous case (see expression 4.3). Since we need this time to get a quintic curve, we will substitute the constant factor $m$ in $z_{1}$ and $z_{2}$ we introduced in (4.4) by the square root of a quadratic real polynomial

$$
m(t)=\left(m_{0} B_{0}^{2}(t)+m_{1} B_{1}^{2}(t)+m_{2} B_{2}^{2}(t)\right) .
$$

Therefore

$$
\begin{aligned}
& z_{1}(t)=m(t)^{\frac{1}{2}}(a+(t-1)(a+b)), \\
& z_{2}(t)=m(t)^{\frac{1}{2}} t,
\end{aligned}
$$

and the hodograph can be computed from Eq. 2.3.

Now, we can proceed analogously to what we did for the cubical case. The deduction is skipped. Parameters $a, b, m_{0}, m_{1}, m_{2}$ can be determined from the Hermite conditions as follows:

(1) Compute $a=\frac{x_{v}+\mathbf{i} y_{v}}{1-z_{v}}, m_{2}=\frac{1}{2} r_{v}\left(1-z_{v}\right)$.

(2) Put

$$
b=\frac{1}{3 m_{0}+4 m_{1}+3 m_{2}}\left(2\left(m_{0}+3 m_{1}+6 m_{2}\right) a-30 r_{q}\left(x_{q}+\mathbf{i} y_{q}\right)\right) .
$$

(3) If $1-z_{v}-2 m_{0} \neq 0$, then

$$
m_{1}=\frac{4 m_{0}^{2}-r_{v}(1-z)^{2}-12 m_{0}\left(1-r_{v}\left(1-z_{v}\right)-z_{v}+5 r_{q}\left(x_{v} x_{q}+y_{v} y_{q}-\left(1-z_{v}\right) z_{q}\right)\right)}{6\left(1-z_{v}-2 m_{0}\right)} \text {. }
$$


(a) Solve in the variable $m_{0}$ the quartic equation $|b|^{2} m_{0}=1$, after substitution of the other parameters.

(4) If $1-z_{v}-2 m_{0}=0$, then take $m_{0}=\frac{1}{2} \frac{r_{v}}{1-z_{v}}=m_{2}$.

(a) Solve in the variable $m_{1}$ the quadratic equation $|b|^{2} m_{0}=1$, after substitution of the other parameters.

(5) Once all possible solutions have been obtained, those for which the factor $m(t)=\left(m_{0} B_{0}^{2}(t)+m_{1} B_{1}^{2}(t)+m_{2} B_{2}^{2}(t)\right)$ is not strictly positive for $t \in[0,1]$ should be rejected. We will call admissible solutions those with a strictly positive $m(t)$.

5.2. Using general helical $\mathbf{P H}$ interpolants. The second possibility is the use of general helical $\mathrm{PH}$ interpolants. In the monotone helical $\mathrm{PH}$ curves, we have used a degree 1 Bézier parametrization of a straight line and then we have multiplied the complex functions $z_{1}$ and $z_{2}$ by a common nonconstant factor in order to obtain the desired degree for the hodograph. For general helical PH curves, and under the light of the Exemple 2.1.2 and subsection 3.1, specially Eq. (3.1), what we will use is a degree 2 rational parametrization of a straight line and then to multiply the functions $z_{1}$ and $z_{2}$ by a common constant factor.

Any degree 2 rational Bézier curve is determined by a control polygon, $\left\{P_{0}, P_{1}, P_{2}\right\}$, and a set of weights $\left\{w_{0}, w_{1}, 1\right\}$. If we want to obtain a parametrization of a straight line, then the control polygon must satisfy $P_{1}=(1-\lambda) P_{0}+\lambda P_{2}$ for some $\lambda \in \mathbb{R}$.

Since we want a rational curve passing through $\infty$ when $t=0$, we will use the parameter $\frac{1}{t}$ instead of $t$ :

$$
\frac{B_{0}^{2}\left(\frac{1}{t}\right) \omega_{0} P_{0}+B_{1}^{2}\left(\frac{1}{t}\right) \omega_{1} P_{1}+B_{2}^{2}\left(\frac{1}{t}\right) P_{2}}{B_{0}^{2}\left(\frac{1}{t}\right) \omega_{0}+B_{1}^{2}\left(\frac{1}{t}\right) \omega_{1}+B_{2}^{2}\left(\frac{1}{t}\right)}=\frac{(t-1)^{2} \omega_{0} P_{0}+2(t-1) \omega_{1} P_{1}+P_{2}}{(t-1)^{2} \omega_{0}+2(t-1) \omega_{1}+1}
$$

The denominator, $(t-1)^{2} \omega_{0}+2(t-1) \omega_{1}+1$, vanishes at $t=0$ if and only if $\omega_{0}=2 \omega_{1}-1$.

Finally, in order to simplify computations we write:

$$
P_{0}=a+b, \quad P_{1}=(1-\lambda) P_{0}+\lambda P_{2}, \quad P_{2}=a,
$$

where $a, b \in \mathbb{C}$ and $\lambda \in \mathbb{R}$ and weights

$$
\{2 \omega-1, \omega, 1\},
$$

where $\omega \in \mathbb{R}$.

Remark 2. Two values of the weight $\omega$ are singular. If $\omega=1$ then all weights are 1 and the initial rational Bézier curve becomes just a Bézier curve. If $\omega=\frac{1}{2}$ then the initial degree 2 rational Bézier curve is in fact a degree 1 rational curve, and we have then a monotone helical PH, but not a general helical PH. 
According to these conventions

$$
\begin{aligned}
& z_{1}(t)=m(a t(2-t-2(1-t) \omega)+b(1-t)(-1+t+2(\lambda-t) \omega)), \\
& z_{2}(t)=m(t(2-t-2(1-t) \omega)),
\end{aligned}
$$

with $m \in \mathbb{C}$, and the hodograph can be computed from Eq. 2.3

Parameters $a, b, m, \lambda, \omega$ can be determined from the Hermite conditions as follows:

(1) Compute $a=\frac{x_{v}+\mathbf{i} y_{v}}{1-z_{v}}, m^{2}=\frac{1}{2} r_{v}\left(1-z_{v}\right)$.

(2) Write $\lambda=\frac{1-\mu}{2 \omega}$.

(3) Put

$$
b=\frac{30\left(x_{q}+\mathbf{i} y_{q}\right)-4 a m^{2}(8-\omega(7-2 \omega))}{m^{2}(7-5 \mu(3-2 \omega)-2 \omega(9-4 \omega))} .
$$

(4) Solve, with $\mu$ and $\omega$ as unknowns, the system

$\left\{\begin{aligned}|b||m \mu| & =1, \\ (1-2 \omega)^{2}-5 \mu(1-2 \omega) & =2 \mu^{2}\left(\left(1+|a|^{2}\right) m^{2}(8-\omega(7-2 \omega))-5\left(1+3\left(a_{0} x_{v}+a_{1} y_{v}-z_{v}\right)\right) .\right.\end{aligned}\right.$

The final step in the previous algorithm is to decide among the real solutions which is the best. One possibility is to choose the one with least possible energy associated with the rotation-minimizing frame (see section 4.4 in [6]). But let us propose another criterion: to choose curves for which the tangent indicatrix is a simple curve, i.e., such that the tangent indicatrix passes just once through each point of its trace.

Proposition 1. The tangent indicatrix of the general quintic PH helix defined by control points (5.1) and weights (5.2) is a simple curve in the interval $[0,1]$ if and only if

$$
\left.\omega<1, \quad \text { and } \quad \frac{2 \omega(\lambda-1)}{1-2 \omega} \notin\right] 0,1[\text {. }
$$

Recall that cases $\omega=1$ and $\omega=\frac{1}{2}$ (Remark 2) have been removed when dealing with general quintic PH helices.

Proof. The injectivity of the tangent indicatrix $t \rightarrow \overrightarrow{\mathbf{t}}(t)$ with $t \in[0,1]$ is equivalent to the injectivity of the map $t \in[0,1] \rightarrow \frac{z_{1}}{z_{2}}(t) \in \mathbb{C} \cup\{\infty\}$.

By definition, see $(5.3)$, we can write $\frac{z_{1}}{z_{2}}(t)$ as the parametrization of a piece of a straight line

$$
\frac{z_{1}}{z_{2}}(t)=a+\frac{(1-t)(-1+t+2(\lambda-t) \omega)}{t(2-t-2(1-t) \omega)} b .
$$

The injectivity of the map $t \in[0,1] \rightarrow \frac{z_{1}}{z_{2}}(t) \in \mathbb{C} \cup\{\infty\}$ is equivalent to the injectivity of

$$
t \in[0,1] \rightarrow f(t):=\frac{(1-t)(-1+t+2(\lambda-t) \omega)}{t(2-t-2(1-t) \omega)} \in \mathbb{R} \cup\{\infty\} .
$$


To make computations easier, we will study the injectivity of the map

$t \in\left[1,+\infty\left[\cup\{\infty\} \rightarrow g(t):=f\left(\frac{1}{t}\right)=\frac{(1-t)(1-t-2 \omega+2 \lambda t \omega)}{1-2 \omega+2 t(\omega-1)} \in \mathbb{R} \cup\{\infty\}\right.\right.$.

The denominator of $g(t)$ has one real root $t_{0}=\frac{1-2 \omega}{2(1-\omega)}=1-\frac{1}{2(1-\omega)}$. Then, the first necessary condition in order to be $g$ injective in $[1,+\infty[\cup\{\infty\}$ is $\left.t_{0} \notin\right] 1,+\infty\left[\cup\{\infty\}\right.$, or equivalently, $\omega<1$. Note that if $t_{0}=1$ then $g\left(t_{0}\right)=\infty$ and there is no other value of $t$ with $\infty$ as image.

Under this condition, we just need to control injectivity of $g$ in $[1,+\infty[$.

The function $g$ is injective in $\left[1,+\infty\right.$ [ if and only if $g^{\prime}(t) \neq 0$ for any $t \in] 1,+\infty\left[\right.$. Note that the possibility $g^{\prime}(1)=0$ is allowed, but this does not affect the injectivity of $g$ in the whole interval $[1,+\infty[$.

Let us call $x:=\frac{2 \omega(\lambda-1)}{1-2 \omega}$, or equivalently $\lambda:=1+\frac{1}{2}\left(\frac{1}{\omega}-2\right) x$. Therefore, we can write function $g$ as

$$
g(t)=(2 \omega-1) \frac{(1-t)(1+t(x-1))}{1-2 \omega+2 t(\omega-1)} .
$$

It is easy to check that $g^{\prime}(t)=0$ if and only if

$$
(1-x)\left(2(\omega-1) t^{2}+2(1-2 \omega) t+2 \omega\right)+x=0 .
$$

The two roots of this quadratic equation are

$$
t_{i}:=1-\frac{1-x \pm \sqrt{(1-x)(1+(1-2 \omega) x)}}{2(1-\omega)(1-x)}, i:=1,2 .
$$

Let $\Delta=(1-x)(1+(1-2 \omega) x)$. Note that $\Delta \geq 0$ if and only if $\frac{1}{2 \omega-1} \leq x \leq 1$.

Recall that we are interested in showing that if $x \notin] 0,1[$, then there are no extremal points of $g$ for $t \in] 1,+\infty[$, because in such a case, the sign of $g^{\prime}$ will remain unchanged and $\left.g\right|_{[1,+\infty[}$ will be injective.

(1) If $x>1$, then $\Delta<0$ and $g^{\prime}(t) \neq 0$ for any $t \in[1,+\infty[$.

(2) If $x=1$, then the derivative of the function $g$ is

$$
g^{\prime}(t)=\frac{(1-2 \omega)}{(1-2 \omega+2 t(\omega-1))^{2}} .
$$

Therefore, the sign of $g^{\prime}$ in $[1,+\infty$ [ remains unchanged. Moreover, note that condition $\omega<1$ implies that the denominator does not vanishes for $t \in] 1,+\infty[$.

(3) If $x=0$ then it is easy to check that the two roots of $g^{\prime}(t)=0$ are $t_{1}=1$ and $t_{2}=-\frac{\omega}{1-\omega}<0$.

(4) If $x<0$ we have to discuss two cases:

(a) If $\left.\omega \in] \frac{1}{2}, 1\right]$, then $\frac{1}{2 \omega-1} \geq 1>0$. Therefore, $x<\frac{1}{2 \omega-1}$ and then, again $\Delta<0$.

(b) If $\omega<\frac{1}{2}$. In this case $\frac{1}{2 \omega-1}<0$. We split it into two subcases: 
(i) If $x<\frac{1}{2 \omega-1}$, then again $\Delta<0$.

(ii) If $\frac{1}{2 \omega-1} \leq x<0$. In this case, it is just a matter of computation to check that both roots, $t_{1}, t_{2}$ in Eq. (5.5), verify $t_{i}<1$.

Therefore, in all cases, if $x \notin] 0,1[$, then there are no extremal points of $g$ in $] 1,+\infty[$ and we have prooved the sufficiency of conditions 5.4.

Finally, for the necessity, note that if $x \in] 0,1[$, and of course $\omega<1$, then it is easy to check that $\Delta>0$. Indeed, if $\omega<1$ then $1-2 \omega>-1$ and then

$$
(1+(1-2 \omega) x)>1-x .
$$

Therefore, both factors in the definition of $\Delta$ are positive.

The only thing to be checked now is that at least one of the two roots $t_{i}$, $i=1,2$, in Eq. (5.5), verify $t_{i}>1$.

If $x \in] 0,1[$, then the root with the minus sign in the square root can be written as

$$
t_{2}=1+\frac{1}{2(1-\omega)}\left(\sqrt{\frac{1+(1-2 \omega) x}{1-x}}-1\right) .
$$

As we have said before (see Eq. 5.6), the expression inside the square root is greater than 1 . Therefore, $t_{2}>1$.

We will call admissible solutions those for which $\omega$ and $\lambda$ verify conditions (5.4).

Remark 3. The existence of two real roots of the denominator $z_{2}(t)$, one of them $t=0$ and the other $t_{1} \in \mathbb{R}$ implies that the total tangent indicatrix, i.e., when defined in the whole $\mathbb{R}$ and not just in $[0,1]$, is a doubly traced circle. This behavior is already pointed in [6]. The novelty here is the fact that among the solutions one can pick up those for which the tangent indicatrix is a simple curve in $[0,1]$ just by controlling the values of some parameters, without no need of computation of energy integrals. Nevertheless one can not be sure a priori that there is always one and only one of the solutions whose tangent indicatrix is a simple curve ${ }^{2}$. The advantage of the new criterion is the possibility of skipping the computation of the energy in a lot of cases.

\section{EXAMPLES}

The author has been unable to find some statement vaguely similar to Theorem 2, that is, to find some conditions to the existence or not of quintic interpolants in terms of some angles and/or lengths. Instead, let us illustrate with examples some of the possibilities one can find when solving the first order Hermite problem. Computations have been done using Mathematica.

The energy computed along the examples is the rotation-minimizing frame

$$
\mathcal{E}_{\mathrm{RMF}}=\int_{0}^{1} \kappa^{2}(t)\left\|\alpha^{\prime}(t)\right\| d t
$$

\footnotetext{
${ }^{2}$ This has been pointed out by one of the referees.
} 
6.1. Example 1. In [6] the authors work the following example with Hermite data

$$
\mathbf{p}_{0}=(0,0,0), \quad \mathbf{d}_{0}=(1,0,1), \quad \mathbf{p}_{1}=(1,1,1), \quad \mathbf{d}_{1}=(0,1,1) .
$$

Thanks to the combination of a rotation and a change of scale of ratio $\frac{1}{\sqrt{2}}$ the Hermite data can be reduced to

$$
\mathbf{q}_{0}=(0,0,0), \quad \mathbf{v}_{0}=(0,0,1), \quad \mathbf{q}_{1}=\left(\frac{1}{\sqrt{2}}, 0,1\right), \quad \mathbf{v}_{1}=\left(\frac{1}{\sqrt{2}}, \frac{1}{2}, \frac{1}{2}\right) .
$$

Notice that the initial and final directions are unit vectors.

6.1.1. Monotone. There are two admissible solutions

$$
m_{2}=m_{0}=\frac{1}{4}, \quad \text { and } m_{1}=\frac{51}{44} \quad \text { or } m_{1}=\frac{21}{4} .
$$
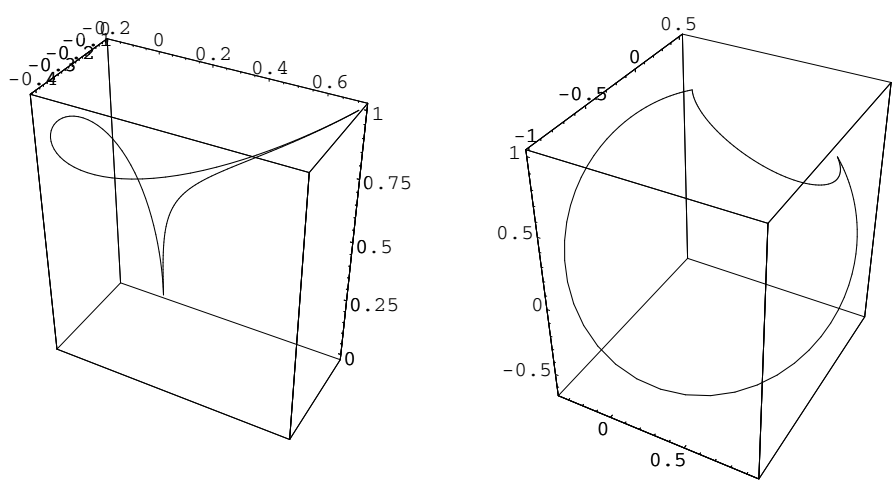

Figure II. The two monotone helical quintic Hermite interpolants for the data $\mathbf{q}_{0}=(0,0,0), \quad \mathbf{v}_{0}=(0,0,1), \quad \mathbf{q}_{1}=\left(\frac{1}{\sqrt{2}}, 0,1\right), \quad \mathbf{v}_{1}=\left(\frac{1}{\sqrt{2}}, \frac{1}{2}, \frac{1}{2}\right)$.

Left: the two helices. Right: their tangent indicatrices.

The ratio curvature over torsion for the solution $m_{1}=\frac{51}{44}$ is equal to $\frac{9}{11 \sqrt{2}}$, approximately 0.578542 . The energy for it is 2.04694. The other solution has a comparatively higher energy, 28.8104 .

6.1.2. General. There is just one admissible solution:

$$
\mu=-1, \quad \omega=-0.10038
$$

This solution is the one given in [6]. The ratio $\frac{\kappa}{\tau}=0.586692$ and its energy 1.80114. So, in this case the general helix is better than the two monotone helices. 

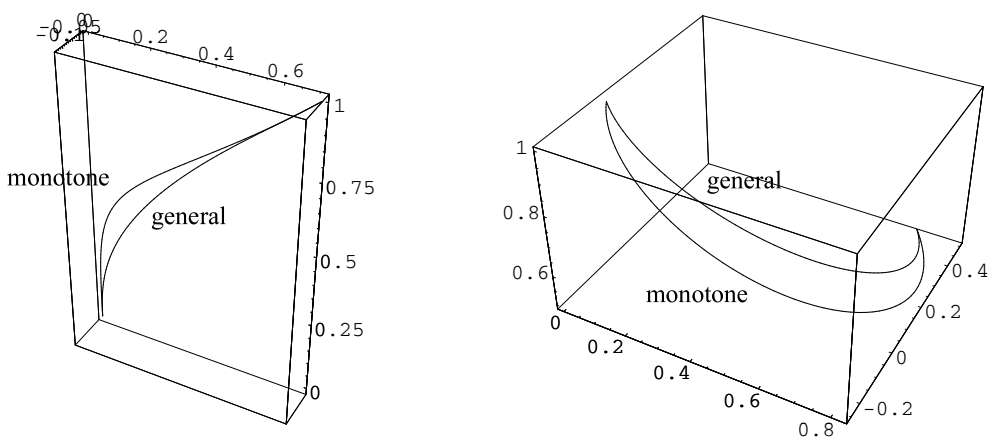

Figure III. Comparison between one of the monotone solution and the general solution for the data

$\mathbf{q}_{0}=(0,0,0), \quad \mathbf{v}_{0}=(0,0,1), \quad \mathbf{q}_{1}=\left(\frac{1}{\sqrt{2}}, 0,1\right), \quad \mathbf{v}_{1}=\left(\frac{1}{\sqrt{2}}, \frac{1}{2}, \frac{1}{2}\right)$.

Left: the two helices. Right: their tangent indicatrices.

6.2. Example 2. In section 4.5 of [6] an example with end derivatives of different norm is also studied:

$\mathbf{p}_{0}=(0,0,0), \quad \mathbf{d}_{0}=(-0.8,0.3,1.2), \quad \mathbf{p}_{1}=(1,1,1), \quad \mathbf{d}_{1}=(0.5,-1.3,-1.0)$.

First, after spatial transformations we can reduce the problem to

$$
\mathbf{q}_{0}=(0,0,0), \quad \mathbf{v}_{0}=(0,0,1),
$$

$\mathbf{q}_{1}=(1.13068,0,0.322581), \quad \mathbf{v}_{1}=(-0.471992,-0.539519,-0.917051)$.

6.2.1. Monotone. Now, there is just one admissible monotone solution:

$$
m_{2}=1.04051, \quad m_{1}=8.29381 \text { and } m_{0}=0.553794 .
$$

The ratio $\frac{\kappa}{\tau}=-5.80019$ and its energy 6.36576 .

6.2.2. General. There is just one admissible solution:

$$
\mu=-0.389398, \text { and } \omega=-0.166546 .
$$

The ratio $\frac{\kappa}{\tau}=-4.97734$ and its energy 12.4304. So, this time, the monotone solution is now better.
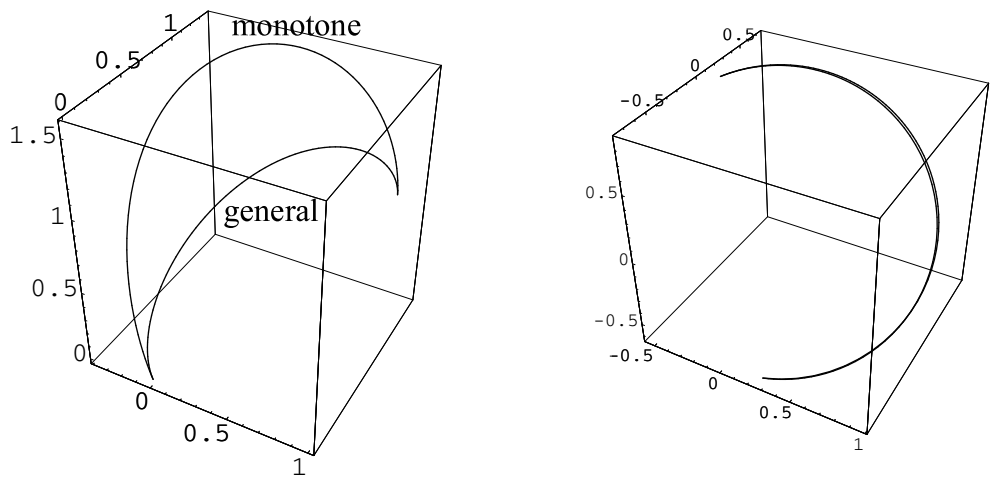
Figure IV. Comparison between the monotone solution and the general solution for the data in Example 2. Left: the two helices. Right: their tangent indicatrices.

6.3. Example 3. Let us work a final example proposed in [6] (section 4.4, Fig. 2):

$\mathbf{p}_{0}=(0,0,0), \mathbf{d}_{0}=(0.4,-1.5,-1.2), \mathbf{p}_{1}=(1,1,1), \mathbf{d}_{1}=(-1.2,-0.6,-1.2)$.

Thanks to the scale factor $2 \sqrt{\frac{5}{77}}$, a rotation along the $y$-axis of angle $-\arccos \frac{3}{\sqrt{10}}$ and a rotation along the $x$-axis of angle $-\arccos \left(-4 \sqrt{\frac{2}{77}}\right)$ the problem is reduced to

$$
\begin{aligned}
\mathbf{p}_{0}=(0,0,0), & \mathbf{d}_{0}=(0,0,1), \\
\mathbf{p}_{1}=(0.64466,-0.08214,-0.59740), & \mathbf{d}_{1}=(-0.77359,-0.09856,0.48312) .
\end{aligned}
$$

6.3.1. Monotone. Now, there are no admissible monotone solutions.

6.3.2. General. Again, there is just one admissible general solution.

This time let us be more explicit. For such a data, there are four real solutions for $\mu$ and $\omega$ :

$\begin{array}{ccccc}\text { solution } & \mu & \omega & \frac{2 \omega(\lambda-1)}{1-2 \omega}=1-\frac{\mu}{1-2 \omega} & \text { energy } \\ 1 & -0.98780 & 7.64178 & & 92.020 \\ 2 & 1.54688 & 7.19549 & & 121.974 \\ 3 & -0.98136 & -4.14178 & 1.10571 & 31.741 \\ 4 & 0.61442 & -3.69549 & 0.92678 & 223.867\end{array}$

Only the third solution verifies conditions (5.4). Moreover, it is the one with less energy. (See Figures V and VI.)

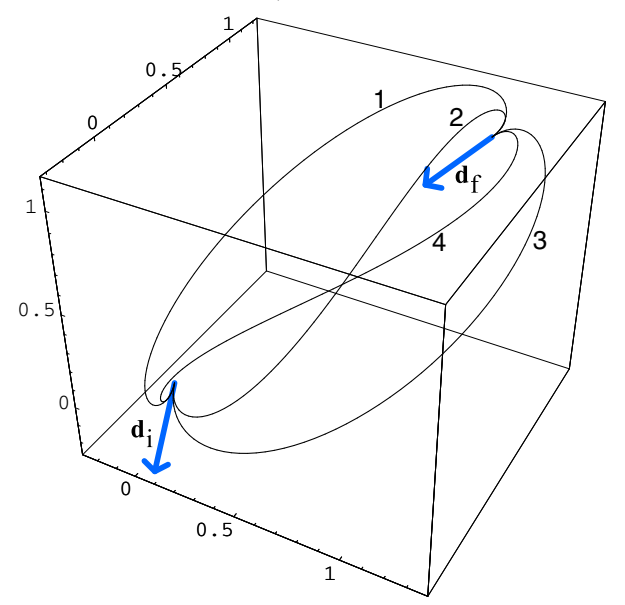

Figure V. The four solutions initially obtained for Hermite data $\mathbf{p}_{0}=$ $(0,0,0), \mathbf{d}_{0}=(0.4,-1.5,-1.2), \mathbf{p}_{1}=(1,1,1), \mathbf{d}_{1}=(-1.2,-0.6,-1.2)$. 


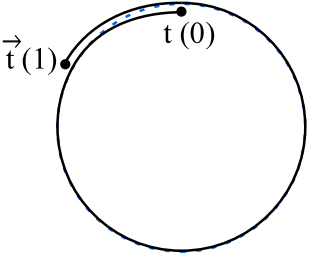

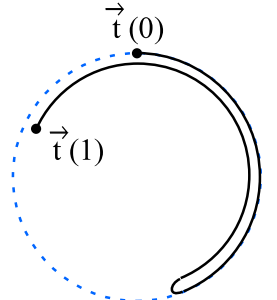

2

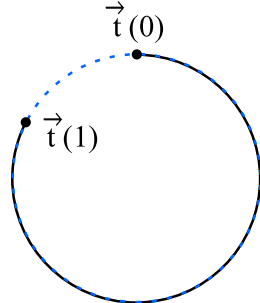

3

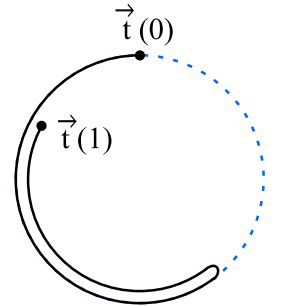

4

Figure VI. Schematic plot of the tangent indicatrices of the four solutions.

Only solution number 3 is a simple curve. In solutions 2 and 4 a point with stationary tangent vector is present.

\section{Conclusions}

The simplifications proposed along this paper seem to be useful both from a theoretical and constructive point of view.

In all the examples, here in the paper or in previous computer computations, we always have found just one admissible general solution. Whereas for monotone helices we have shown examples with two, one or none solutions. Moreover, the energy of the monotone helix can be even better than the energy of the general solution.

Let us finally say that this simplified approach should be also useful to undertake related problems like the second order Hermite problem or the bending problem for helical curves.

Acknowledgement The author wants to express his sincere gratitude to the referees. Their careful reports have contributed to improve the contents of the paper.

\section{REFERENCES}

[1] M. Berger, Geometry. I, Universitext, Springer-Verlag, Berlin, (1987), Translated from the French by M. Cole and S. Levy.

[2] J.V. Beltran, J. Monterde, A characterization of quintic helices, J. of Computational and Applied Mathematics, 206, 116-121 (2007).

[3] R. Dietz, J. Hoschek, Jüttler, B.: An algebraic approach to curves and surfaces on the sphere and on other quadrics, Computer Aided Geometric Design, 10, 211-229 (1993).

[4] H. I. Choi, D. S. Lee, H. P. Moon, Clifford algebra, spin representation, and rational parametrization of curves and surfaces, Advances in Computational Mathematics, 17, $5-48,(2002)$.

[5] R. T. Farouki, Pythagorean-Hodograph Curves, in Handbook of Computer Aided Geometric Design, Edited by G. Farin, J. Hoschek, M.-S. Kim, North Holland, 405-427 (2002).

[6] R. T. Farouki, Ch. Y. Han, C. Manni, A. Sestini, Characterization and construction of helical polynomial space curves, Journal of Computational and Applied Mathematics, 162, 365-392 (2004).

[7] B. Jüttler, C. Mäurer, Cubic Pythagorean hodograph spline curves and applications to sweep surface modeling, Comput. Aided Design, 31, 73-83 (1999). 
[8] F. Pelosi, R. T. Farouki, C. Manni, A. Sestini, Geometric Hermite interpolation by spatial Pythagorean-hodograph cubics, Advances in Computational Mathematics, 22, 325-352 (2005).

[9] J. R. Silvester, Geometry. Ancient and Modern, Oxford University Press, (2001).

Dep. de Geometria i Topologia, Universitat de València, Avd. Vicent AndrÉs Estellés, 1, E-46100-Burjassot (VAlÈncia), Spain

E-mail address: monterde@uv.es 